\title{
Proper motion measurements as indicators of binarity in open clusters
}

\author{
E. Bica and C. Bonatto \\ Universidade Federal do Rio Grande do Sul, Instituto de Física, CP 15051, Porto Alegre 91501-970, RS, Brazil \\ e-mail: charles@if.ufrgs.br
}

Received 16 September 2004 / Accepted 25 October 2004

\begin{abstract}
We analyze 9 open clusters with ages in the range $70 \mathrm{Myr}$ to $3.2 \mathrm{Gyr}$ using UCAC2 proper motion data and 2MASS photometry, which allows us to reach stellar masses down to $\approx 0.7 M_{\odot}$. We employ in this work an approach in which the background proper motion contribution is statistically subtracted in order to obtain the cluster's intrinsic proper motion distribution. For each cluster we consider the projected velocity distributions in the core and off-core regions separately. In the projected velocity distribution of all sample clusters we find a well-defined low-velocity peak, as well as an excess in the number of stars at larger velocities. The low-velocity peak is accounted for by the random motion of the single stars, while the high-velocity excess can be attributed to the large velocity changes produced by a significant fraction of unresolved binaries in a cluster. We derive kinematic parameters of the single-star distribution, in particular the projected velocity dispersion. The relatively large velocity dispersions derived in this work may reflect the non-virialized state of the clusters. Based on the relative number of high-velocity (binary) and single stars, we inferred for the sample clusters unresolved binary fractions in the range $15 \% \leq f_{\text {bin }} \leq 54 \%$, for both core and off-core regions. Stars with a projected velocity exceeding the maximum reached by the single-star distribution are identified in 2MASS $J \times(J-H)$ colour-magnitude diagrams. The asymmetry observed in the distribution of these stars around the main sequence is consistent with models of main-sequence widening resulting from unresolved binaries combined with 2MASS photometric uncertainties. The present results suggest that care must be taken when applying proper-motion filters to sort out members, especially binaries in a star cluster. This paper shows that proper motions turn out to be a useful tool for identifying high-velocity stars as unresolved binary cluster members, and as a consequence, map and quantify the binary component in colour-magnitude diagrams.
\end{abstract}

Key words. Galaxy: open clusters and associations: general

\section{Introduction}

Binaries play a vital rôle in the dynamics of star clusters since, as a result of the combined effect of high star density and low velocity dispersion, the energy locked up in binary binding energy may be comparable to, or even exceed that of the whole cluster (Hut 1996). Consequently, changes in binary properties along the normal stellar evolution will affect the dynamical evolution of the cluster as a whole, particularly the old, more massive clusters. In this context, the identification and quantification of the binary fraction is fundamental for understanding the dynamical evolution of star clusters.

Open clusters are known to contain significant fractions of the stellar content in the form of multiple systems, binaries in particular (e.g. Montgomery et al. 1993; von Hippel \& Sarajedini 1998). And, as a consequence of the dynamical evolution in open clusters, the fraction of multiple systems both increases and tends to concentrate in the central regions, thus changing the initial spatial distribution of stars (Takahashi \& Portegies Zwart 2000).
Observationally, the main effects of a significant fraction of unresolved binaries in the central parts of a star cluster are expected to be: $(i)$ the production of large deviations in the cluster's proper motion distribution with respect to the single-star component, since the resulting velocities may be much larger for the binary motions; (ii) the underestimate of the number of low-mass stars with respect to higher-mass stars; and (iii) the widening of the main-sequence (MS) towards higher magnitudes in a colour-magnitude diagram (CMD). Point $(i)$ is the fundamental issue addressed for the first time in the present paper. As a consequence of (ii), the observed central luminosity (or mass) function turns out to be flatter than that of the actual, resolved stellar distribution. In addition, the resulting mass-function changes will depend both on the binary fraction and MS mass range (e.g. Kroupa et al. 1991, and references therein).

Rather than consider the proper motion components of individual stars, which might exclude unresolved binaries as nonmember stars, we apply in this work a statistical analysis. Our approach is to compare the proper motion (modulus) distribution of the stars in the cluster field with that of the offset field 
Table 1. General data on the target clusters.

\begin{tabular}{|c|c|c|c|c|c|c|c|c|c|c|}
\hline \multirow[b]{2}{*}{ Cluster } & \multicolumn{5}{|c|}{ WEBDA } & \multicolumn{4}{|c|}{ Present work } & \multirow[b]{2}{*}{$\begin{array}{l}R_{\lim } \\
(\mathrm{pc})\end{array}$} \\
\hline & $\begin{array}{c}\alpha(2000) \\
(\mathrm{hms})\end{array}$ & $\begin{array}{c}\delta(2000) \\
\left({ }^{\circ}, \prime \prime\right)\end{array}$ & $\begin{array}{c}\text { Age } \\
(\mathrm{Myr})\end{array}$ & $E(B-V)$ & $\begin{array}{c}d_{\odot} \\
(\mathrm{kpc})\end{array}$ & $\begin{array}{l}\text { Age } \\
(\mathrm{Myr})\end{array}$ & $E(B-V)$ & $\begin{array}{c}d_{\odot} \\
(\mathrm{kpc})\end{array}$ & $\begin{array}{l}R_{\text {core }} \\
(\mathrm{pc})\end{array}$ & \\
\hline (1) & (2) & (3) & (4) & (5) & (6) & (7) & (8) & (9) & (10) & (11) \\
\hline M26 & $18: 45: 18$ & $-09: 23: 00$ & 85 & 0.59 & 1.60 & $70 \pm 10$ & $0.42 \pm 0.03$ & $1.57 \pm 0.07$ & $0.9 \pm 0.1$ & $7.8 \pm 0.9$ \\
\hline NGC 2287 & 06:46:01 & $-20: 45: 24$ & 243 & 0.03 & 0.69 & $160 \pm 10$ & 0.00 & $0.76 \pm 0.04$ & $1.4 \pm 0.2$ & $8.6 \pm 0.4$ \\
\hline M48 & $08: 13: 43$ & $-05: 45: 00$ & 360 & 0.03 & 0.77 & $360 \pm 40$ & 0.00 & $0.76 \pm 0.03$ & $0.9 \pm 0.2$ & $8.9 \pm 0.4$ \\
\hline M93 & $07: 44: 30$ & $-23: 51: 24$ & 387 & 0.05 & 1.04 & $400 \pm 50$ & 0.00 & $1.05 \pm 0.04$ & $0.6 \pm 0.1$ & $5.5 \pm 0.6$ \\
\hline NGC 5822 & $15: 04: 21$ & $-54: 23: 48$ & 662 & 0.15 & 0.92 & $1000 \pm 100$ & 0.00 & $0.72 \pm 0.03$ & $0.9 \pm 0.1$ & $8.5 \pm 0.4$ \\
\hline NGC 6208 & $16: 49: 28$ & $-53: 43: 42$ & 1170 & 0.21 & 0.94 & $1300 \pm 100$ & $0.21 \pm 0.03$ & $1.31 \pm 0.06$ & $0.9 \pm 0.2$ & $6.5 \pm 0.8$ \\
\hline NGC 3680 & $11: 25: 38$ & $-43: 14: 36$ & 1200 & 0.07 & 0.94 & $1600 \pm 100$ & 0.00 & $1.05 \pm 0.05$ & $0.6 \pm 0.1$ & $7.0 \pm 0.6$ \\
\hline IC 4651 & $17: 24: 49$ & $-49: 56: 00$ & 1140 & 0.12 & 0.89 & $1800 \pm 200$ & 0.00 & $0.91 \pm 0.04$ & $0.9 \pm 0.1$ & $5.9 \pm 0.3$ \\
\hline M 67 & $08: 51: 18$ & $+11: 48: 00$ & 2560 & 0.06 & 0.91 & $3200 \pm 100$ & 0.00 & $0.87 \pm 0.09$ & $1.2 \pm 0.1$ & $8.8 \pm 0.9$ \\
\hline
\end{tabular}

Notes. The 2MASS colour excess for M 26 is $E(J-H)=0.14$, and for NGC $6208 E(J-H)=0.07$. Uncertainties in Cols. (7)-(9) are derived from the isochrone fit.

stars. Thus, the presence of unresolved binaries should be indicated by significant deviations in the cluster's proper motion modulus distribution.

In the present work we employ $J$ and $H 2 \mathrm{MASS}^{1}$ data, which provide photometry for stars in a large spatial area in the direction of a cluster, and proper motion data from $\mathrm{UCAC}^{2}$ in the same field.

This paper is organized as follows. In Sect. 2 we provide general data on the target clusters. 2MASS photometry, CMDs and structural parameters for the clusters are presented in Sect. 3. The proper motion analysis is presented in Sect. 4. Finally, concluding remarks are given in Sect. 5.

\section{The target clusters}

The open clusters selected for the present analysis are M 26 (NGC 6694), NGC 2287, M 48 (NGC 2548), M 93 (NGC 2447), NGC 5822, NGC 6208, NGC 3680, IC 4651 and M 67 (NGC 2682). These clusters span a wide range in age, which is important, since the binary fraction in a cluster is expected to increase as a function of dynamical evolution (age). General data for the above open clusters are given in Table 1. The data in Cols. 2-6 have been obtained from the WEBDA open cluster database (Mermilliod 1996) ${ }^{3}$. In Cols. 7-11 we list parameters derived from the present work using 2MASS data (Sect. 3).

In previous studies binaries have already been detected in some of the above clusters. Stetson (1981) indicated that NGC 5822 contains a high percentage of binary stars and several blue stragglers. Levato \& Malaroda (1979) found that most of the B 8-A0 stars in NGC 2287 are spectroscopic

${ }^{1}$ The Two Micron All Sky Survey, All Sky data release (Skrutskie et al. 1997), available at http://www.ipac. caltech.edu/2mass/ releases/allsky/

2 The Second US Naval Observatory CCD Astrograph Catalog (Zacharias et al. 2003), available at http:// vizier.u-strasbg.fr/viz-bin/VizieR?-source=UCAC2

${ }^{3}$ http://obswww. unige.ch/webda binaries. Nordström et al. (1997) identified several single and binary cluster members in NGC 3680 . With respect to IC 4651, Meibom et al. (2002) found that $37 \%$ of the 19 red giants are spectroscopic binaries with periods of up to 5000 days, while $52 \%$ of the $67 \mathrm{MS}$ stars are binaries with periods less than 1000 days. Montgomery et al. (1993) found that at least 38\% of the stars in M 67 are binaries. Mermilliod \& Mayor (1989) have found a percentage of $25-33 \%$ of binaries in M 93 .

\section{The 2MASS photometry}

2MASS has proved to be a useful tool to explore various aspects related to open clusters, e.g. Bica et al. (2003, 2004). We derive parameters for the present cluster sample from $J$ and $H$ 2MASS photometry. The VizieR tool ${ }^{4}$ was used to extract stars in a circular area centered on the coordinates given in Table 1. In order to maximize the statistical significance and representativity of background star counts, we used stars extracted in an external annulus as offset field.

\subsection{Colour-magnitude diagrams}

In Fig. 1 we present the $J \times(J-H)$ CMDs for the open clusters dealt with in this paper. To maximize cluster membership probability, the stars included in the CMDs correspond to a region with radius $2 \times R_{\text {core }}$ (Sect. 3.2). Cluster parameters were derived by fitting solar metallicity Padova isochrones (Girardi et al. 2002) computed with the 2MASS $J$ and $H$ filters $^{5}$, to the observed CMDs. The 2MASS transmission filters produced isochrones very similar to the Johnson ones, with differences of at most 0.01 in $(J-H)$ (Bonatto et al. 2004), see also Grocholski \& Sarajedini (2003) for an analysis of combined optical/near-infrared CMDs. For reddening and absorption transformations we use $R_{V}=3.2$, and the relations

\footnotetext{
4 http://vizier.u-strasbg.fr/viz-bin/

VizieR?-source $=$ II $/ 246$

5 Available to download at http://pleiadi.pd.astro.it/ isoc_photsys.01/isoc_photsys.01.html
} 


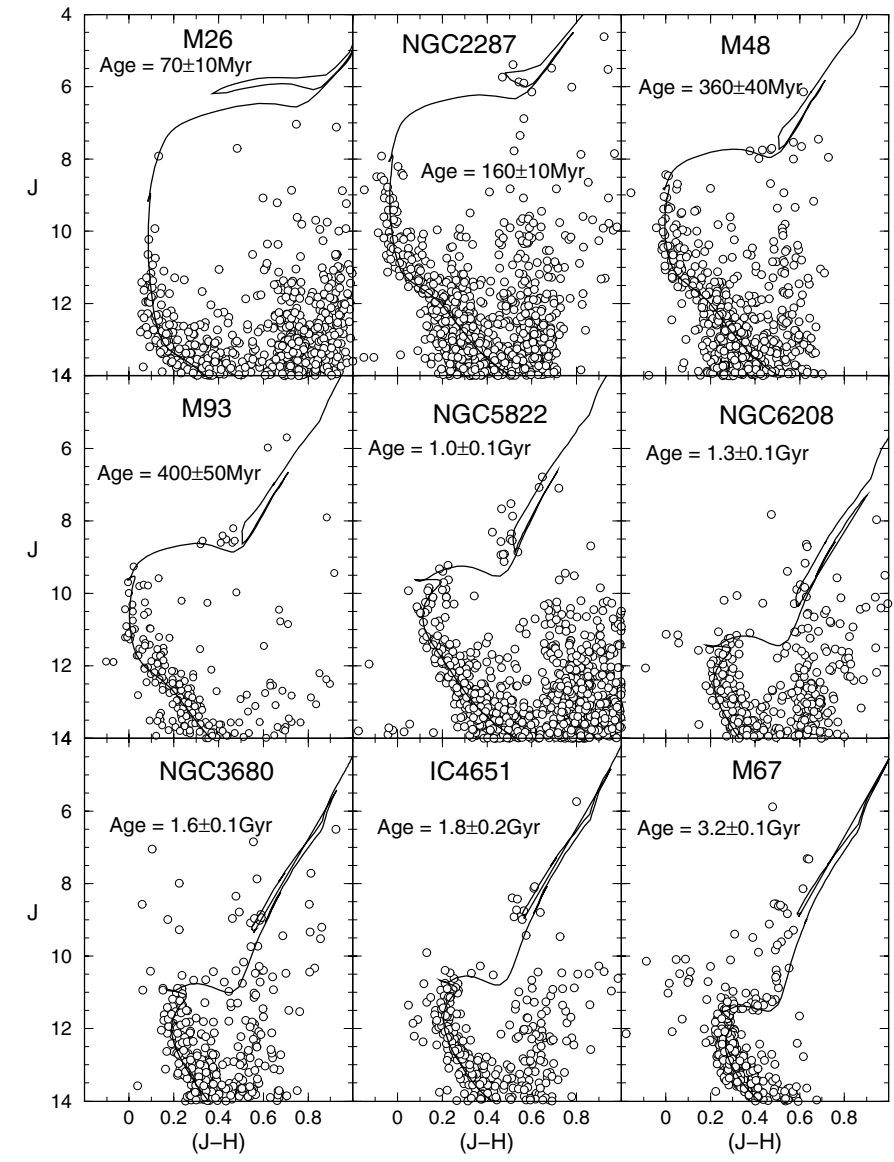

Fig. 1. $J \times(J-H)$ CMDs for the present open clusters. In each panel, the region shown corresponds to a radius $r=2 \times R_{\text {core }}$. The solid line shows the best-fit Padova isochrone. The derived age is indicated in each panel.

$A_{J}=0.276 \times A_{\mathrm{V}}$ and $E(J-H)=0.33 \times E(B-V)$, following Dutra et al. (2002) and references therein. The resulting age, colour excess and distance to the Sun, are given respectively in Cols. 7-9 of Table 1. Except for M 26 and NGC 6208, no amount of reddening was detected for the clusters.

For most clusters, the optical value for $E(B-V)$ in Table 1 is larger than that inferred from the near-infrared. However, we note that $E(B-V)=3 \times E(J-H)$, so these near-infrared bands are in fact less sensitive to reddening determinations. In previous studies we already noticed the need for less equivalent reddening in the near-infrared than what was expected from the optical, e.g. the case of M 67 (Bonatto \& Bica 2003). The excesses $E(J-H)$ are derived from the overall isochrone fit to the CMD sequences. Besides, for several of the present clusters, deep CMDs are studied. As can be seen in the CMDs of Fig. 1, there is not much freedom for variations in $E(J-H)$ measurements. Basically, all values of $E(B-V)$ smaller than 0.06 are null in the near-infrared. In the case of IC 4651 (Fig. 1), the WEBDA value $E(B-V)=0.12$ would prohibitively shift the isochrone too far into the red.

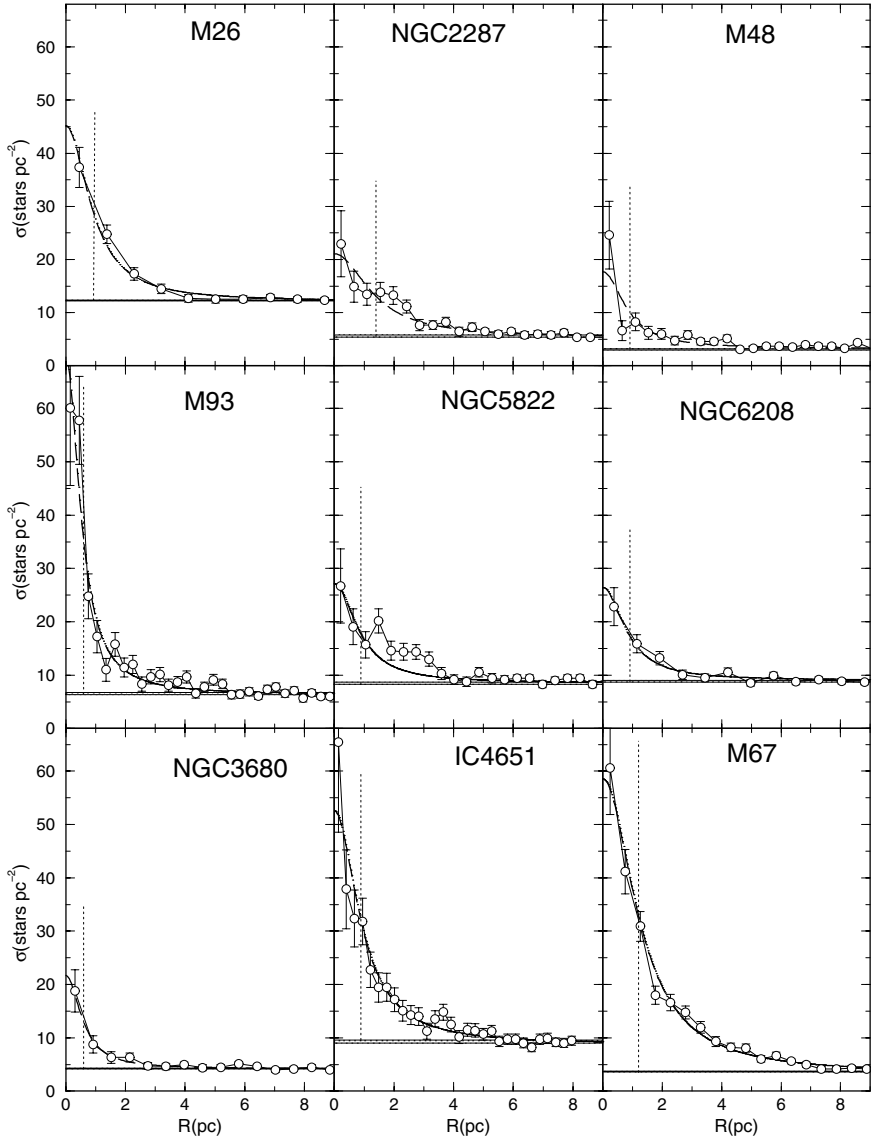

Fig. 2. Projected radial distributions of stars. For each cluster, the average background level is shown as a narrow shaded area; $1 \sigma$ Poisson errors are also shown. The dashed lines show the two-parameter King model fitted to the radial distributions of stars. In each panel, the core radius is indicated as a dotted line. For a better comparison, all panels are plotted on the same scale.

\subsection{Cluster structure}

Structural parameters for the clusters have been derived by means of the radial distribution of stars, defined as the projected number of stars per area around the cluster center. Before counting stars, we applied colour filters to both cluster and corresponding offset field, in order to take account of the contamination of the Galaxy. This procedure has been previously applied in the analysis of the open clusters M 67 (Bonatto \& Bica 2003), NGC 188 (Bonatto et al. 2004) and NGC 3680 and NGC 2180 (Bonatto et al. 2004). The radial distribution was determined by counting stars inside concentric annuli with a step of $1.0^{\prime}$ in radius. The background contribution level corresponds to the average number of stars included in the external annulus (offset field).

The resulting projected radial density profiles for the present clusters are shown in Fig. 2. For a better comparison between clusters, the radii on the abscissas have been scaled from arcmin to parsecs using the distances derived in Sect. 3.1. The statistical significance of each profile is reflected by the $1 \sigma$ Poisson error bars.

First-order structural parameters for each cluster are derived by fitting the two-parameter King (1966a) surface density 
profile to the background-subtracted radial distribution of stars. The two-parameter King model essentially describes the central region of normal clusters (King 1966b; Trager et al. 1995). The fits were performed using a non-linear least-squares fit routine which uses the errors as weights. The best-fit solutions are shown in Fig. 2 as dashed lines, and the resulting core radii are given in Col. 10 of Table 1. Considering the profile fluctuations with respect to the background level, we can define a limiting radius $\left(R_{\text {lim }}\right)$ for each cluster. For regions beyond $R_{\lim }$ the null-contrast between cluster and background star density would produce exceedingly large Poisson errors and consequently, meaningless results. Thus, for practical purposes, we can consider that most of the cluster's stars are contained within $R_{\text {lim }}$. The limiting radii are given in Col. 11 of Table 1 .

\section{Proper motion analysis}

Proper motion components in right ascension and declination for the cluster stars have been obtained from UCAC2. Rather than work separately with proper motion components, we employ in the present work the projected velocity on the sky $v_{\mathrm{p}}=\sqrt{\mu_{\alpha}^{2} \times \cos (\delta)^{2}+\mu_{\delta}^{2}}$, which corresponds to the modulus of the proper motion components. Although affected by the systemic motion, asymmetries in the proper motion distribution of a cluster may yield information on the internal kinematics. To be consistent with the 2MASS analysis, we extracted from UCAC2 proper motions for each cluster inside the same area as that used to extract 2MASS photometry. Since the UCAC2 catalogue also includes the 2MASS photometry, we verified that the correspondence of the two catalogues is nearly complete for $J \leq 14.5$. This is important since for the interpretation of proper motion data in the context of determining the physical nature of an object one must include faint stars as well.

\subsection{M67, a test case}

Because of its relatively high Galactic latitude $\left(b \approx 32^{\circ}\right)$ the field of M 67 (NGC 2682) presents little background contamination (Fig. 1). Thus, M 67 is an ideal cluster to examine the probable association of proper motion with the presence of binary stars. In this sense, M 67 is an interesting cluster because it is known to contain a significant fraction of unresolved binaries (Montgomery et al. 1993). In Fig. 3 we show the $J \times(J-H)$ CMDs of the central region of M 67 (left panel) and the corresponding (same area) offset field (right panel). Stars with 2MASS detections are indicated by open circles, while those with proper motion determined by UCAC2 are indicated by dots. For stars with $J \leq 14.5$ both catalogues are essentially complete. We also show in both panels the colour filter used to isolate most M67 stars from the background contamination. Probable M67 blue stragglers (at $10 \leq J \leq 11$ and $0.0 \leq(J-H) \leq 0.3)$ have also been excluded from the analysis. The relatively large number of stars in M 67 also allows us to study spatial variations in the proper motion distribution. Thus, the present analysis will be applied to both core and offcore regions.

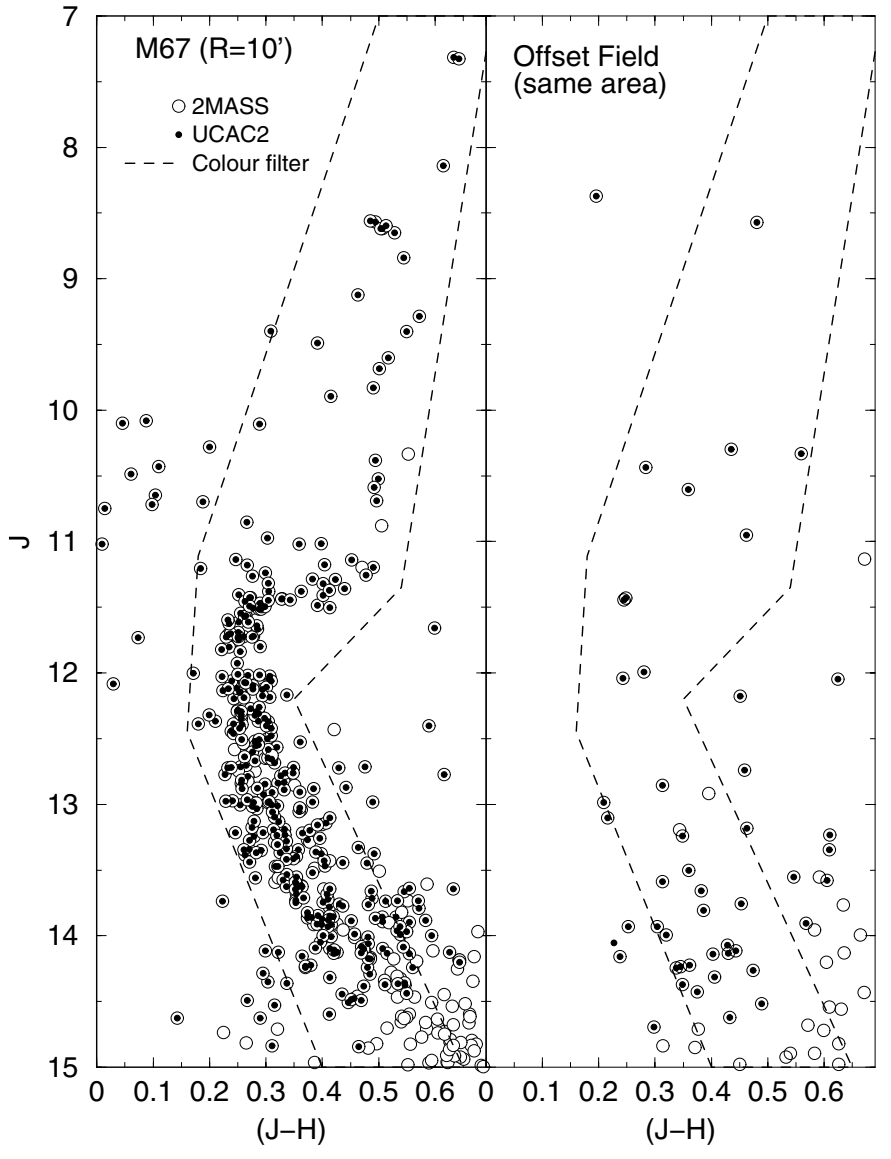

Fig. 3. Correspondence between the 2MASS photometry and UCAC2 data for M67. Both catalogues are nearly complete for $J<14.5$. The colour filter is shown as dashed lines. Left panel: central $\left(R<10^{\prime} \approx\right.$ $2.5 \mathrm{pc}$ ) region; right panel: offset field (same area).

Although small, the contamination by Galactic stars of the CMD of M67 must be taken into account to isolate the intrinsic proper motion distribution in this cluster. We study the properties of the proper motions in M 67 according to the following procedure. First we apply the colour filter shown in Fig. 3 to both cluster and offset field stars. The filtering process takes into account most of the background (mostly Galaxy disk stars) contamination, leaving only a residual contamination which will be dealt with by the offset field. Next we build histograms with the number of stars in velocity bins of 2 mas $\mathrm{yr}^{-1}$ width, both for the cluster and the offset field. This procedure is applied to both core $(0.0 \leq R(\mathrm{pc}) \leq 1.2)$ and offcore $(1.2 \leq R(\mathrm{pc}) \leq 8.8)$ regions. Then, the offset field histogram is scaled to match the projected area of both regions. Finally, the subtraction of the offset field histogram from those of the core and off-core regions produces the intrinsic proper motion distribution in M67. This procedure is illustrated in Fig. 4 for the proper motion components in right ascension $\left(\mu_{\alpha}\right)$ and declination $\left(\mu_{\delta}\right)$. As expected for a self-gravitating system, the proper motion distributions in the core and off-core regions of M 67 present systematic deviations with respect to the offset field distribution.

Significant deviations in proper motion with respect to a Gaussian distribution are seen in both $\mu_{\alpha}$ and $\mu_{\delta}$ components. 


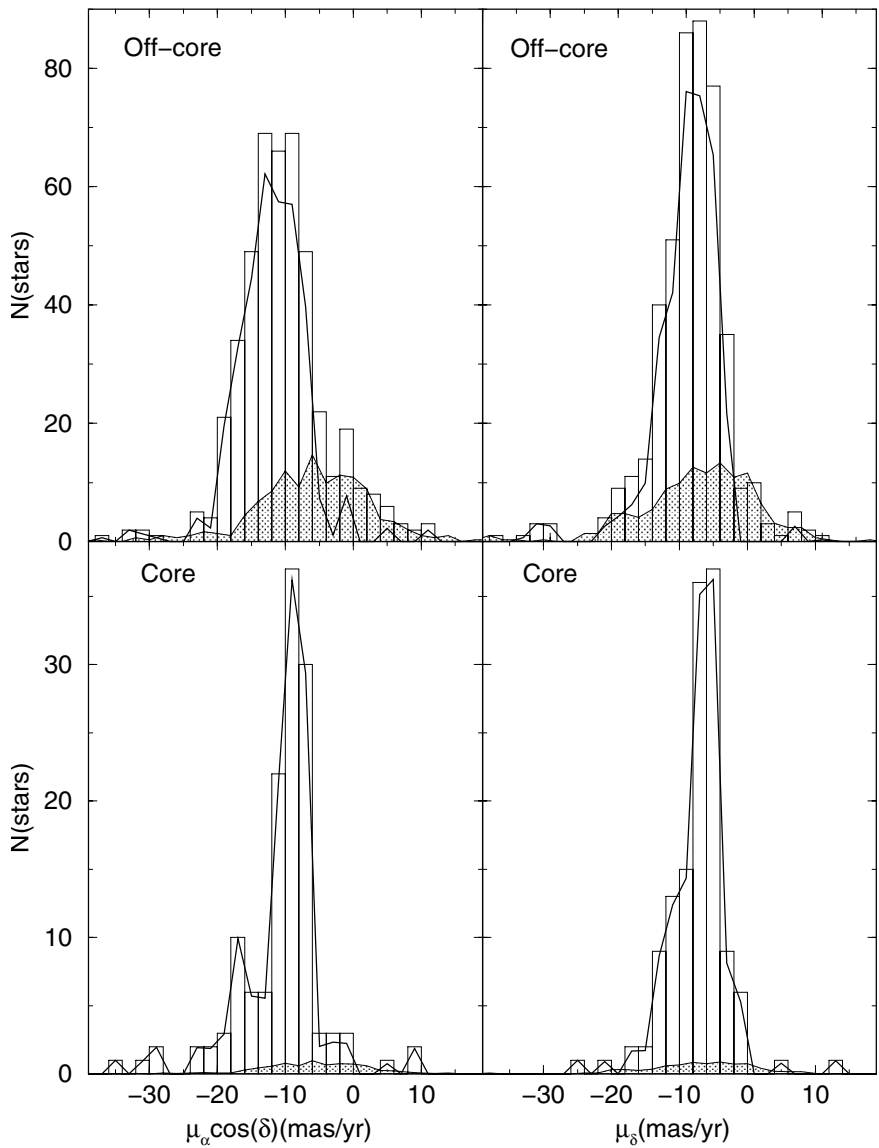

Fig. 4. Proper motion components in the core (bottom panels) and offcore (top panels) regions of M 67. Histograms in the left panels show the number of stars per velocity bin for the $\mu_{\alpha}$ component; $\mu_{\delta}$ components are in the right panels. The shaded areas correspond to the offset field (same area) contribution. The solid line represents the intrinsic proper motion distribution in each region.

Considering the offset field contribution, the excesses may be accounted for by a component of high-velocity stars inside M 67. In order to characterize these deviations in terms of highvelocity stars, we hereafter use the projected velocity $v_{\mathrm{p}}$. The units mas $\mathrm{yr}^{-1}$ have been transformed to $\mathrm{km} \mathrm{s}^{-1}$ using the distances listed in Col. 9 of Table 1. The above procedure is illustrated in Fig. 5, in which the left panels show the projected velocity histograms both for the cluster regions and offsetfield, while the right panels display the intrinsic, offset fieldsubtracted, projected velocity distribution in M67. The statistical significance of this procedure can be estimated from the resulting Poisson error bars.

The projected velocity distributions both in the core and off-core regions of M 67 present conspicuous low-velocity and high-velocity peaks. The low-velocity peaks can be accounted for by the random motion of the single stars, superimposed on the cluster's systemic motion. On the other hand, the highvelocity peaks, which considering the error bars represent real excesses, may be produced by unresolved binary systems, in which the presence of a secondary changes appreciably the velocity of the primary star. Kinematical parameters for the cluster are derived by fitting Gaussian functions $N \propto \exp -\frac{\left(v-v_{0}\right)^{2}}{2 \sigma_{v}^{2}}$ to

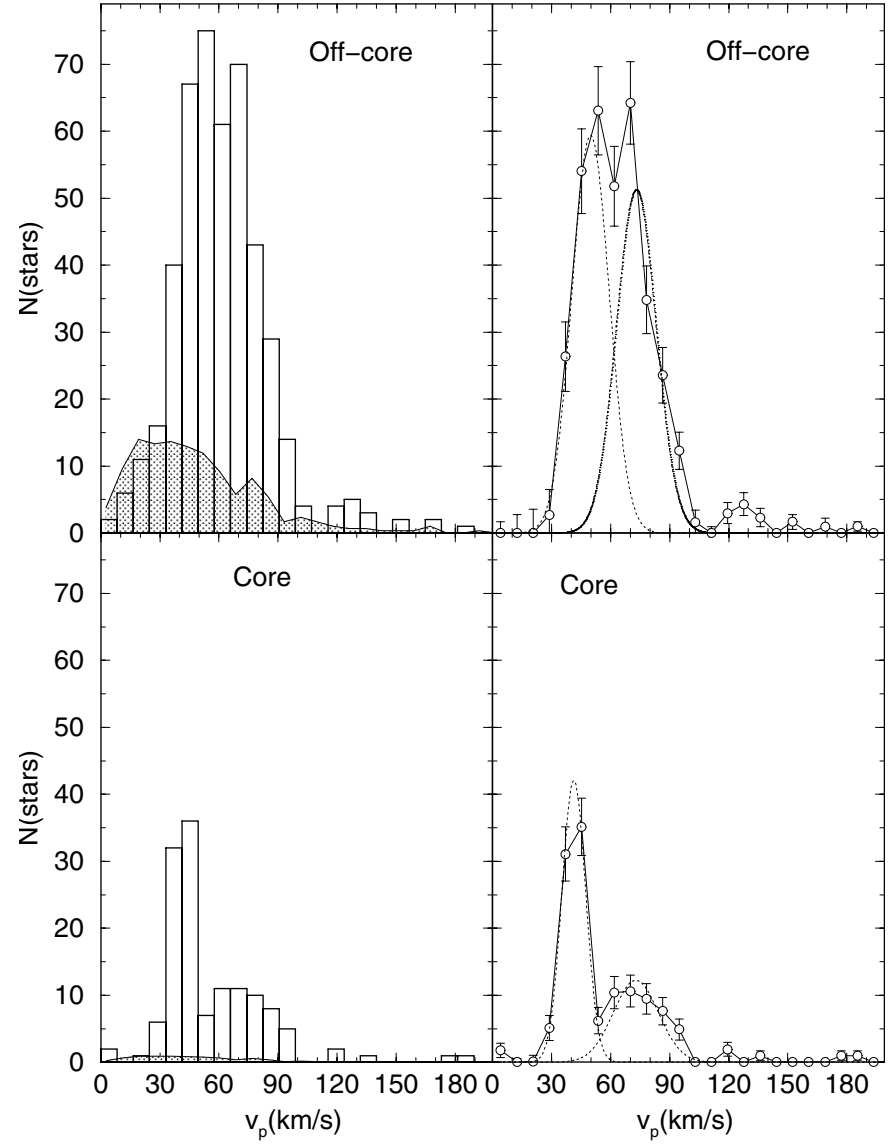

Fig. 5. Distribution of projected velocity of stars in M67. Histograms in the left panels show the number of stars per velocity bin in the core (bottom panel) and off-core regions (top panel). The shaded area corresponds to the offset field (same area) contribution. Right panels: projected velocity distribution. Dotted lines: Gaussian fits to the distributions. Note an additional high-velocity peak centered at $v_{\mathrm{p}} \approx 130 \mathrm{~km} \mathrm{~s}^{-1}$ in the off-core profile of M 67 .

the $v_{\mathrm{p}}$ distributions, where $v_{0}$ is the peak average velocity and $\sigma_{v}$ is the projected velocity dispersion. We found for the core region a low-velocity peak centered at $v_{0}=41.3 \pm 0.7 \mathrm{~km} \mathrm{~s}^{-1}$, with a velocity dispersion $\sigma_{v}=6.0 \pm 0.6 \mathrm{~km} \mathrm{~s}^{-1}$. The highvelocity peak, with smaller amplitude, is centered at $v_{0}=$ $72.8 \pm 2.3 \mathrm{~km} \mathrm{~s}^{-1}$, with $\sigma_{v}=11.5 \pm 1.5 \mathrm{~km} \mathrm{~s}^{-1}$. A similar situation is observed in the off-core region, with the low-velocity peak at $v_{0}=49.8 \pm 1.8 \mathrm{~km} \mathrm{~s}^{-1}$, with $\sigma_{v} \approx 7.5 \pm 2.3 \mathrm{~km} \mathrm{~s}^{-1}$, and the high-velocity one at $v_{0} \approx 73.0 \pm 2.2 \mathrm{~km} \mathrm{~s}^{-1}$, with $\sigma_{v} \approx 10.1 \pm 0.7 \mathrm{~km} \mathrm{~s}^{-1}$. The above fits are shown in the right panels of Fig. 5 as dotted lines. Within the uncertainties, $\sigma_{v}$ for the low-velocity peak is the same, both in the core and offcore regions. The same conclusion applies to the high-velocity peaks in both regions.

Considering the $v_{\mathrm{p}}$ distribution in the core region, stars with velocities larger than $70 \mathrm{~km} \mathrm{~s}^{-1}$ can be considered to have deviant proper motions with respect to the single-star distribution. We identify and plot these deviant stars in the CMD of the core region in Fig. 6, in which the $3.2 \mathrm{Gyr}$ isochrone is also plotted for clarity. The high-velocity stars are distributed along the MS with an asymmetry towards the right side of the isochrone. They occupy the locus encompassed by the 


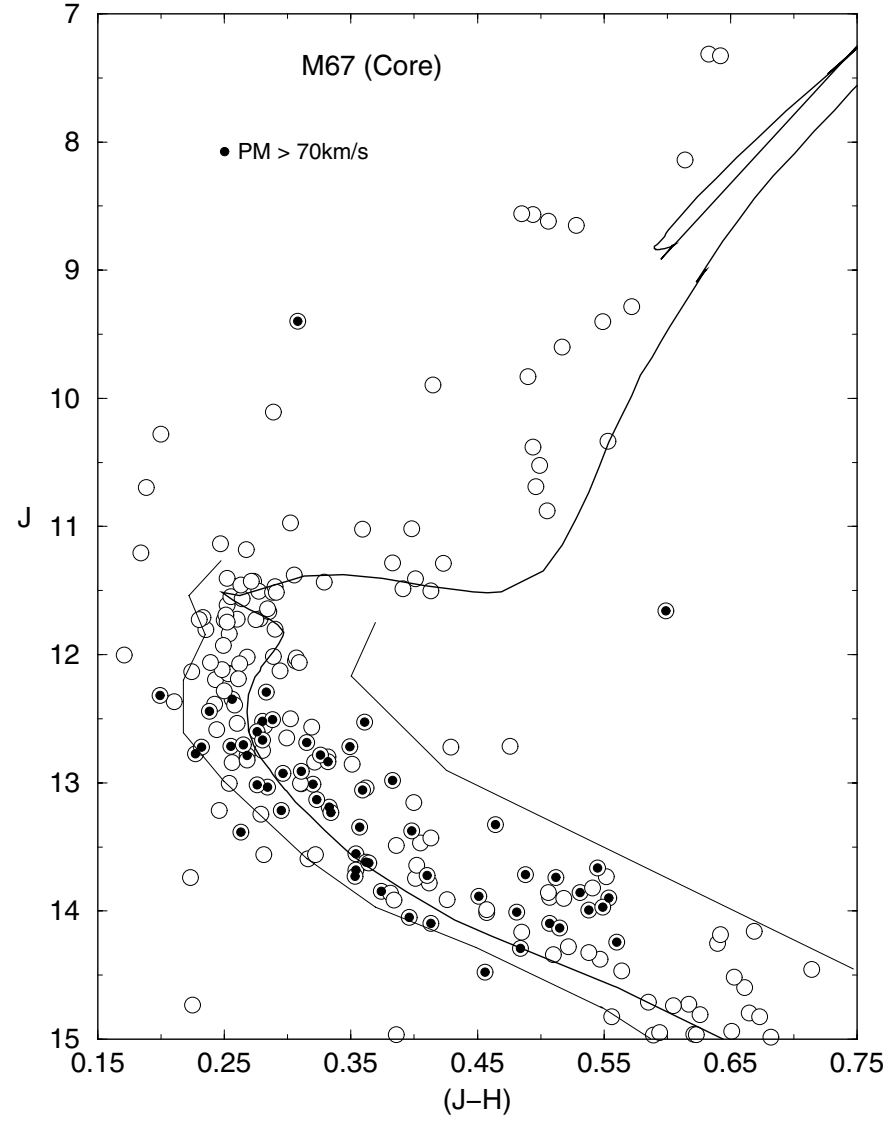

Fig. 6. Stars with projected velocity larger than $70 \mathrm{~km} \mathrm{~s}^{-1}$ (filled circles) are superimposed on the CMD of M 67. The thin solid lines mark the borders of the CMD region occupied by unresolved binaries combined to the 2MASS photometric uncertainties. The $3.2 \mathrm{Gyr}$ isochrone is also shown for clarity.

colour-magnitude limits expected for binary stars combined with the 2MASS photometric uncertainties (thin-solid lines in Fig. 6).

To simulate the effects of unresolved binaries on the 2MASS CMD, we first generate a population of single stars according to a standard $(\chi=1.35)$ Salpeter MF distribution. To be consistent with the 2MASS CMD of M 67 we used single stars with masses lower than the respective turnoff. According to a pre-defined fraction of CMD binaries, we randomly select stars from the original distribution and build the specified number of binaries. No mass or luminosity bias is introduced in this process (Kroupa 2001; Mazeh et al. 2003) and, consequently the probability of a star being selected as a binary member depends only on the number frequency of its mass. In order to obtain the maximum spread in $(J-H)$ colour spanned by the binary distribution we use a binary fraction of $100 \%$, which means that all objects in the CMD are unresolved binary systems. We also include in the simulation the effects of the 2MASS photometric uncertainties in $J$ and $H$. In 2MASS photometry, the average photometric uncertainty $(\epsilon)$ for a given magnitude can be obtained following $\epsilon_{J}=$ $0.023+4.78 \times 10^{-9} \mathrm{e}^{(J / 0.976)}$ and $\epsilon_{H}=0.025+1.92 \times 10^{-8} \mathrm{e}^{(H / 0.998)}$ (Bonatto et al. 2004). For each $J$ and $H$ magnitude, the photometric error is obtained by randomly selecting a value in the interval $\left(-\epsilon_{J, H} \leq \epsilon \leq+\epsilon_{J, H}\right)$, assuming that $\epsilon$ follows a normal

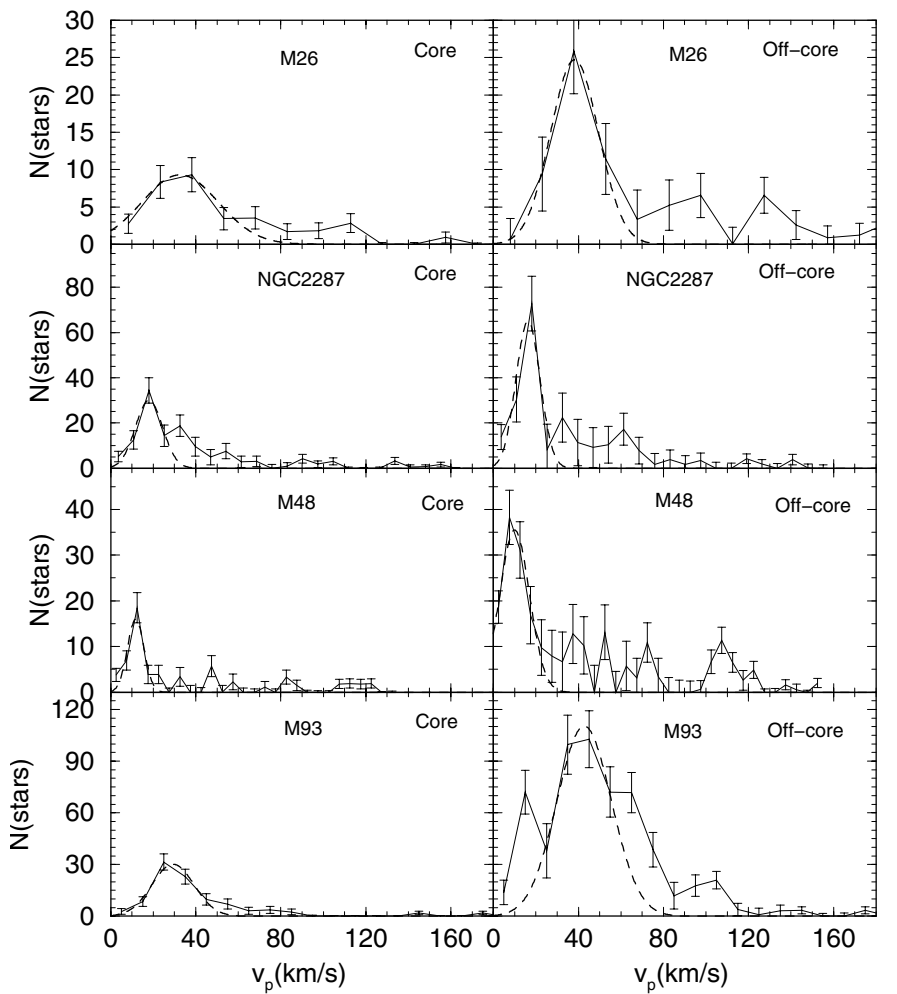

Fig. 7. Projected velocity distributions in the core (left panels) and off-core (right panels) regions. The dashed curves represent a fit to the single-star distributions, according to the parameters in Table 2.

distribution curve. Finally, for each single-mass star and binary in the CMD, uncertainties are assigned to the $J$ and $H$ magnitudes as described above. The resulting maximum colour spreads are indicated in Fig. 6. The high-velocity stars in Fig. 6 do not reach the red border of the simulation. Consequently, the binary fraction must be lower than $100 \%$.

Under the assumption that the low-velocity peak essentially represents the velocity distribution of the single stars, and that the high-velocity excess is caused by unresolved binaries, the binary fraction $\left(f_{\text {bin }}\right)$ in a cluster can be estimated by dividing the number of high-velocity stars by the total number of stars. Thus, in the core region of M67, we find $f_{\text {bin }}=39 \pm 16 \%$, which agrees with the fraction derived by Montgomery et al. (1993). In the off-core region we find $f_{\text {bin }}=45 \pm 12 \%$ which, within the uncertainties, agrees with the binary fraction in the core.

\subsection{The remaining sample}

The remaining clusters have been analyzed in a similar way as M 67, and the corresponding projected velocity distributions for the core and off-core regions are shown in Figs. 7 and 8. Clearly, the low-velocity peak is present in both core and offcore regions in all clusters, with varying central (systemic) velocity and velocity dispersion. This is reflected in the parameters of the Gaussian fits to the low-velocity peaks, which are summarized in Table 2. We include in Table 2 the maximum velocity reached in the single-star distribution, $v_{\text {cut }}$. The high-velocity excess is conspicuously present in most clusters 
Table 2. Properties of the single-star distribution and binaries.

\begin{tabular}{|c|c|c|c|c|c|c|c|c|c|}
\hline \multirow[b]{2}{*}{ Cluster } & \multicolumn{4}{|c|}{ Off-core } & \multirow[b]{2}{*}{$\begin{array}{c}v_{\text {cut }} \\
\left(\mathrm{km} \mathrm{s}^{-1}\right) \\
(6)\end{array}$} & \multicolumn{4}{|c|}{ Core } \\
\hline & $\begin{array}{c}m_{\text {low }} \\
\left(M_{\odot}\right) \\
(2)\end{array}$ & $\begin{array}{c}v_{0} \\
\left(\mathrm{~km} \mathrm{~s}^{-1}\right) \\
(3)\end{array}$ & $\begin{array}{c}\sigma_{v} \\
\left(\mathrm{~km} \mathrm{~s}^{-1}\right) \\
(4)\end{array}$ & $\begin{array}{l}f_{\text {bin }} \\
(\%) \\
(5)\end{array}$ & & $\begin{array}{c}v_{0} \\
\left(\mathrm{~km} \mathrm{~s}^{-1}\right) \\
(7)\end{array}$ & $\begin{array}{c}\sigma_{v} \\
\left(\mathrm{~km} \mathrm{~s}^{-1}\right) \\
(8)\end{array}$ & $\begin{array}{l}f_{\text {bin }} \\
(\%) \\
(9)\end{array}$ & $\begin{array}{c}v_{\text {cut }} \\
\left(\mathrm{km} \mathrm{s}^{-1}\right) \\
(10)\end{array}$ \\
\hline M26 & 1.1 & $38.2 \pm 1.0$ & $11.3 \pm 0.8$ & $39 \pm 21$ & 70 & $32.6 \pm 3.6$ & $18.0 \pm 3.8$ & $18 \pm 12$ & 80 \\
\hline NGC 2287 & 0.6 & $16.3 \pm 2.5$ & $5.7 \pm 2.2$ & $43 \pm 59$ & 35 & $18.0 \pm 1.8$ & $6.4 \pm 1.7$ & $48 \pm 45$ & 40 \\
\hline M 48 & 0.6 & $10.0 \pm 1.5$ & $7.0 \pm 1.8$ & $47 \pm 21$ & 35 & $11.7 \pm 0.8$ & $3.9 \pm 0.8$ & $48 \pm 23$ & 25 \\
\hline M93 & 0.8 & $42.7 \pm 1.2$ & $12.8 \pm 1.6$ & $38 \pm 17$ & 85 & $29.3 \pm 1.3$ & $9.9 \pm 1.2$ & $21 \pm 9$ & 55 \\
\hline NGC 5822 & 0.6 & $41.1 \pm 1.3$ & $8.2 \pm 1.1$ & $16 \pm 10$ & 60 & $43.2 \pm 1.4$ & $5.3 \pm 1.1$ & $16 \pm 8$ & 70 \\
\hline NGC 6208 & 0.8 & $23.0 \pm 2.3$ & $11.0 \pm 2.0$ & $34 \pm 22$ & 60 & $32.1 \pm 1.9$ & $10.1 \pm 1.3$ & $54 \pm 30$ & 60 \\
\hline NGC 3680 & 0.8 & $29.4 \pm 1.8$ & $12.0 \pm 1.5$ & $20 \pm 11$ & 65 & $34.4 \pm 0.4$ & $6.7 \pm 0.3$ & $25 \pm 5$ & 55 \\
\hline IC 4651 & 0.7 & $19.9 \pm 2.3$ & $7.2 \pm 2.1$ & $46 \pm 50$ & 45 & $14.8 \pm 0.5$ & $6.5 \pm 0.5$ & $50 \pm 11$ & 35 \\
\hline M 67 & 0.7 & $49.8 \pm 1.8$ & $7.5 \pm 2.3$ & $45 \pm 12$ & 90 & $41.3 \pm 0.7$ & $6.0 \pm 0.6$ & $39 \pm 16$ & 70 \\
\hline
\end{tabular}

Notes. $m_{\text {low }}$ is the stellar low-mass end reached in this work; $v_{0}$ and $\sigma_{v}$ have been obtained from the Gaussian fit to the low-velocity distribution; $f_{\text {bin }}$ is the binary fraction; $v_{\text {cut }}$ is the maximum velocity reached by the single-star distribution.

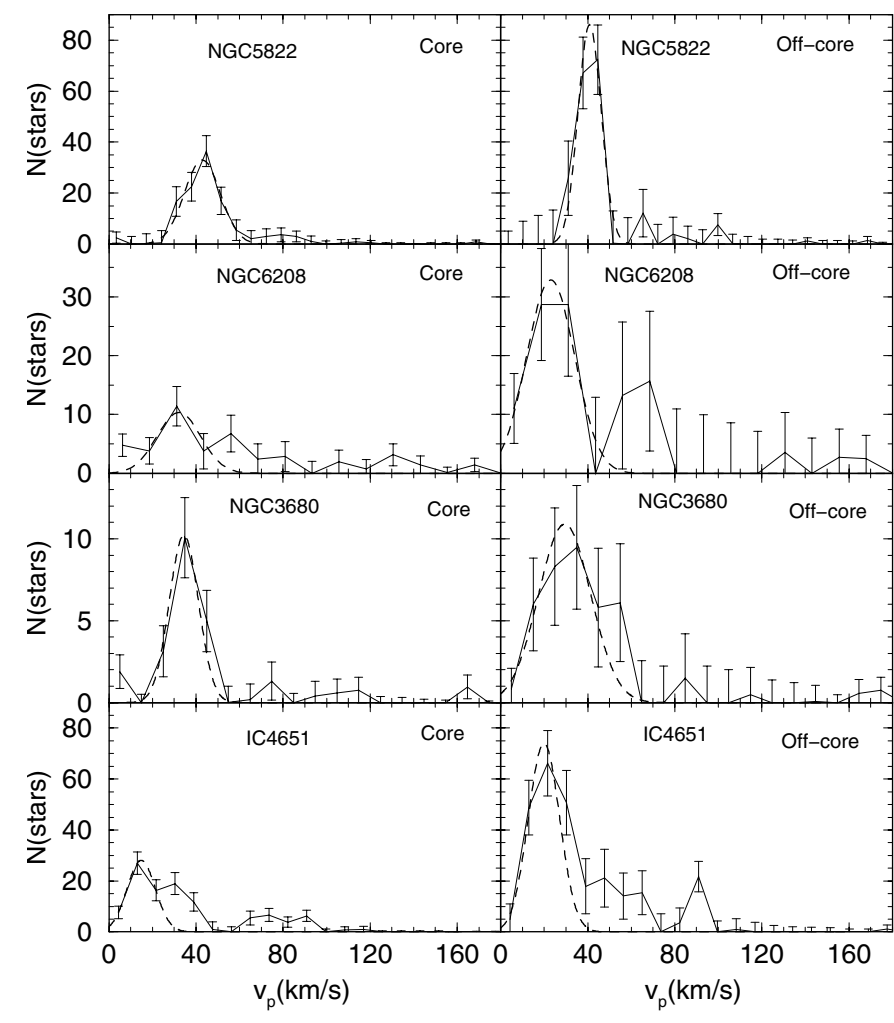

Fig. 8. Same as Fig. 7 for the remaining clusters.

as well, the exceptions being NGC 3680 and NGC 5822 (core and off-core regions), and M 48 and M 93 (core region). The large error bars present in the profiles of the latter clusters, which reflect a relatively small number of stars, make the excess marginal. Gaussian fits have not been applied to the highvelocity excess in the remaining clusters because of the less well defined profiles. Besides, as a consequence of the large error bars the resulting binary fractions contain significant uncertainties. Within the uncertainties, the average binary fraction in the core, $\left\langle f_{\text {bin }}\right\rangle=35 \pm 15 \%$, turns out to be the same as in the off-core region, $\left\langle f_{\text {bin }}\right\rangle=36 \pm 11 \%$ (standard deviation from the average). The same is valid for the average velocity dispersions in the core, $\left\langle\sigma_{v}\right\rangle=8 \pm 4 \mathrm{~km} \mathrm{~s}^{-1}$, and off-core regions, $\left\langle\sigma_{v}\right\rangle=9 \pm 2 \mathrm{~km} \mathrm{~s}^{-1}$. The high-velocity stars (according to Col. 10 of Table 2) that are present in the core region of each of the remaining clusters are identified and plotted on the corresponding CMDs in Fig. 9. For each cluster we simulated the combined effect of binaries and photometric errors, following the approach applied to M67 (Sect. 4.1). The resulting limiting lines are shown in the figure. In most clusters the asymmetry in colour presented by the high-velocity stars with respect to the corresponding isochrone is similar to that displayed by the core of M 67 (Fig. 6), bearing in mind that M 67 is more populous. The observed asymmetries in the CMD distributions support the scenario of unresolved binaries, as further indicated by the present proper motion study.

In the CMD of some clusters, a nearly vertical red sequence shows up (e.g. IC 4651) which we suspect to be solar neighbourhood high-velocity low-mass stars. From their $(J-H)$ colours, we infer them to be $\mathrm{K}$ and $\mathrm{M}$ dwarfs. Similar to the case of M67, most of the high-velocity stars do fall within the red border of the binary simulation, except in M26 and NGC 6208 which are much affected by red stars.

According to the virial theorem, the dynamical mass for a gravitationally bound, spherically symmetric and isotropic cluster is given by $M=3 \sigma^{2} R / G$, where $\sigma$ is the velocity dispersion and $R$ is the effective gravitational radius. As a first-order approximation, we assume $R=R_{\text {core }}$ and $\sigma=$ $\sigma_{v}$ (core). For the range of core radii (Table 1) and velocity dispersions (Table 2) found in the present cluster sample the implied dynamical masses would be in the range $(1-4) \times$ $10^{4} M_{\odot}$. For M 26, the youngest cluster in our sample with the largest $\sigma_{v}$, a mass would result of $\sim 2 \times 10^{5} M_{\odot}$. These mass values turn out to be exceedingly high for open clusters, since the old $(\approx 7 \mathrm{Gyr})$ and very populous open cluster NGC 188 has an estimated mass of $\approx 4 \times 10^{3} M_{\odot}$, as determined from different methods (Bonatto et al. 2004, and references therein). The present clusters are clearly not as populous as NGC 188. 


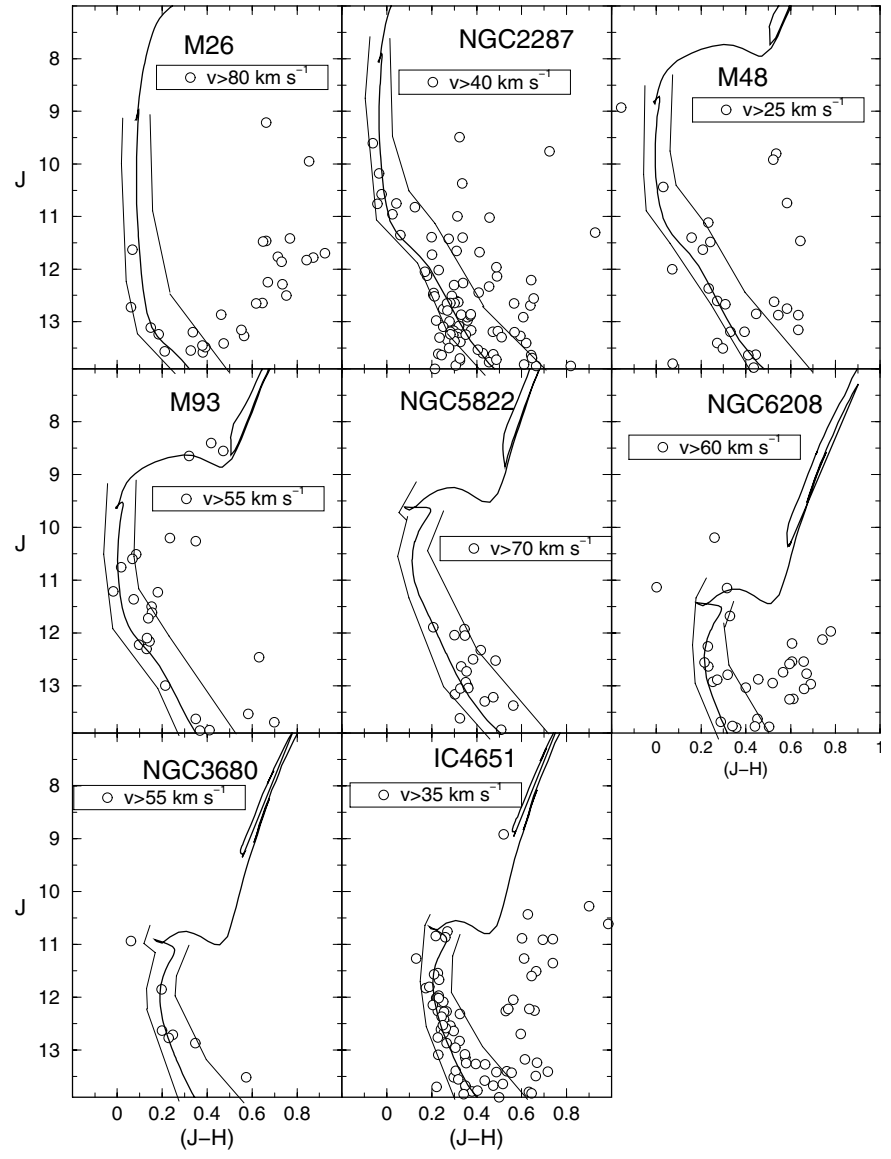

Fig. 9. Distribution of the high-velocity stars in the core region of the remaining clusters. The minimum value for a high-velocity star $\left(v_{\text {cut }}\right)$ is indicated in each panel. For ease of visualization, only the isochrone and the borders of the binary+photometric error simulation are shown.

Consequently, the discrepancy in the mass determinations certainly reflects the non-virialization of the clusters.

The present sample contains one cluster (NGC 2287) in common with the clusters studied in Sagar \& Bhatt (1989). Their analysis was based on radial velocity and proper motion measurements of individual stars, restricted to the brighter, higher-mass cluster members. In contrast, the present 2MASS photometry depth allowed us to reach low-mass stars as well, as can be seen in Col. 2 of Table 2 .

Under the first-order assumption of energy equipartition and different mass groups within a cluster, the velocity dispersion would follow the relation $\sigma \sim 1 / \sqrt{\bar{m}}$, where $\bar{m}$ is the average mass of each stellar group. Thus, Sagar \& Bhatt's (1989) results should favour lower velocity dispersions than the present work. Indeed, the velocity dispersions derived in the present work for the core and off-core regions of NGC 2287 are larger than those found by Sagar \& Bhatt (1989) in similar regions, by a factor of $\approx 6$ in the core and $\approx 3.4$ in the off-core region. The significant differences in velocity dispersion in both studies may be accounted for by the larger number of stars, $\approx 357$ - including stars as faint as $J \approx 15$ and mass $m \geq 0.6 M_{\odot}$ - in the present work, as compared to $\approx 65\left(m \geq 1.7 M_{\odot}\right)$ stars in Sagar \& Bhatt's (1989) sample. The large number of stars allowed us to fit the appropriate Gaussian to the background-subtracted projected velocity distribution of NGC 2287 (Fig. 7). Thus, for such a relatively young cluster as NGC 2287, in which the low-mass stars are expected to be far from virialized, the velocity dispersion derived from a large mass range should be larger than that obtained from higher-mass stars. In addition, Sagar \& Bhatt (1989) derive, for the very young, non-virialized open clusters NGC 2669 and NGC 4755, velocity dispersions of the order of $10 \mathrm{~km} \mathrm{~s}^{-1}$. Interestingly, the velocity dispersion in the core of M 26, the youngest cluster in the present sample $(\approx 70 \mathrm{Myr})$, turns out to be the largest, which agrees with strong non-virialization.

\section{Concluding remarks}

In this paper we have analyzed 9 Galactic open clusters, spanning a range of ages from $70 \mathrm{Myr}$ to $3.2 \mathrm{Gyr}$, in terms of UCAC2 proper motion data and 2MASS $J$ and $H$ photometry. Considering the depth of the 2MASS photometry for the stars with UCAC2 data, the stellar low-mass end in the present analysis reached down to $\approx 0.7 M_{\odot}$. Rather than considering individual stars, we applied in this work a statistical approach in which the cluster's intrinsic proper motion distribution was obtained taking into account the background proper motion contribution. Our goals were to infer the intrinsic binary fraction in the clusters and derive kinematic parameters of the single stars, the line-of-sight velocity dispersion in particular. The large velocity dispersions derived in the present work may reflect the non-virialization of the clusters, particularly with respect to the low-mass stellar component. For each cluster we considered separately the projected velocity $\left(v_{\mathrm{p}}=\sqrt{\mu_{\alpha}^{2} \times \cos (\delta)^{2}+\mu_{\delta}^{2}}\right)$ distributions in the core and off-core regions. We found in all clusters $v_{\mathrm{p}}$ distributions which are characterized by a conspicuous low-velocity peak, as well as an excess in the number of stars for larger velocities, in varying proportions for different clusters. The low-velocity peak can be accounted for by the random motion of the single stars superimposed on the cluster's systemic motion. The high-velocity excess is attributed to the significant velocity changes produced by the presence of unresolved binaries in the clusters. The binary fraction is inferred by taking into account the relative number of high-velocity and single stars. We derived a fraction of unresolved binaries in the range $15 \% \leq f_{\text {bin }} \leq 54 \%$, for both core and off-core regions in the sample clusters. We showed that the asymmetry observed in the distribution of high-velocity stars in 2MASS $J \times(J-H)$ CMDs is consistent with models of MS widening resulting from unresolved binaries associated to 2MASS photometric uncertainties.

A possible application of the present method could be in a discussion of the low binary fraction found in the globular cluster M 4 (1-2\%), a surprising result as pointed out by Richer et al. (2004), when compared to previous estimates for globular clusters, which amount to $\leq 10 \%$ (Richer et al. 1997). Richer et al. (2004) applied to M 4 a proper-motion filtering using multi-epoch HST images over 6 years. Possibly in this method a significant fraction of binaries was overlooked because large-proper-motion stars were discarded. 
We conclude that proper motions are a useful tool for identifying high-velocity stars as unresolved binary cluster members, and as a consequence, mapping and quantifying the binary component in CMDs.

Acknowledgements. This publication makes use of data products from the Two Micron All Sky Survey, which is a joint project of the University of Massachusetts and the Infrared Processing and Analysis Center/California Institute of Technology, funded by the National Aeronautics and Space Administration and the National Science Foundation. We also made use of proper motion data from UCAC2 (The Second US Naval Observatory CCD Astrograph Catalog) as well as from the WEBDA open cluster database. We acknowledge support from the Brazilian Institution CNPq.

\section{References}

Bica, E., Bonatto, C. J., \& Dutra, C. M. 2003, A\&A, 405, 991 Bica, E., Bonatto, C. J., \& Dutra, C. M. 2004, A\&A, 422, 555 Bonatto, C. J., Bica, E., \& Santos Jr., J. F. C. 2004, A\&A, submitted Bonatto, C. J., Bica, E., \& Pavani, D. B. 2004, A\&A, in press Bonatto, C., Bica, E., \& Girardi, L. 2004, A\&A, 415, 571

Bonatto, C., \& Bica, E. 2003, A\&A, 405, 525

Dutra, C. M., Santiago, B. X., \& Bica, E. 2002, A\&A, 381, 219

Girardi, L., Bertelli, G., Bressan, A., et al. 2002, A\&A, 391, 195 Grocholski, A. J., \& Sarajedini, A. 2003, MNRAS, 345, 1015 von Hippel, T., \& Sarajedini, A. 1998, AJ, 116, 1789
Hut, P. 1996, in The Origins Evolutions, and Destinies of Binary Stars in Clusters, ASP Conf. Ser., ed. E. F. Milone, \& J.-C. Mermilliod, 90, 391

King, I. 1966a, AJ, 71, 64

King, I. 1966b, AJ, 71, 276

Kroupa, P. 2002, Science, 295, 82

Kroupa, P., Tout, C. A., \& Gilmore, G. 1991, MNRAS, 251, 293

Levato, H., \& Malaroda, S. 1979, PASP, 91, 636

Mazeh, T., Simon, M., Prato, L., Markus, B., \& Zucker, S. 2003, ApJ, 599,1344

Meibom, S., Andersen, J., \& Nordström, B. 2002, A\&A, 386, 187

Mermilliod, J. C. 1996, in The origins, evolution, and destinies of binary stars in clusters, ASP Conf. Ser., 90, 475

Mermilliod, J.-C., \& Mayor, M. 1989, A\&A, 219, 125

Montgomery, K. A., Marschall, L. A., \& Janes, K. A. 1993, AJ, 106, 181

Nordström, B., Andersen, J., \& Andersen, M. I. 1997, A\&A, 322, 460

Richer, H. B., Fahlman, G. G., Ibata, R. A., et al. 1997, ApJ, 484, 741

Richer, H. B., Fahlman, G. G., Brewer, J., et al. 2004, AJ, 127, 2771

Sagar, R., \& Bhatt, H. C. 1989, MNRAS, 236, 865

Skrutskie, M., Schneider, S. E., Stiening, R., et al. 1997, in The Impact of Large Scale Near-IR Sky Surveys, ed. Garzon et al. (Netherlands: Kluwer), 210, 187

Stetson, P. B. 1981, AJ, 86, 1500

Takahashi, K., \& Portegies Zwart, S. F. 2000, ApJ, 535, 759

Trager, S. C., King, I. R., \& Djorgovsky, S. 1995, AJ, 109, 218

Zacharias, N., Urban, S. E., Zacharias, M. I., et al. 2004, AJ, 127, 3043 